\title{
Consideraciones conceptuales para la comprensión de la gestión ambiental en el sector público (Segunda parte)
}

\author{
Conceptual considerations for the \\ understanding of environmental management \\ in the public sector (Second part)
}

Helmut Johnson Madrigal'

Fecha de recepción: 22 de octubre del 2011 Fecha de aprobación: 17 de julio del 2012

Johnson, $\mathrm{H}$. Consideraciones conceptuales para la comprensión de la gestión ambiental en el sector público (Segunda parte) Tecnología en Marcha. Vol. 26, № I. Pág 19-26. 


\section{Palabras clave}

Gestión ambiental, cultura ambiental, sector público.

\section{Key words}

Environmental Management, Environmental Culture, Public Sector.

\section{Resumen}

El alcance de la Gestión Ambiental en el sector público debe entenderse como la interacción de lo técnico-científico con lo social-participativo. El primero, realizando investigación conducente a soluciones alternativas económicamente factibles y ambientalmente viables, con el fin de que el segundo tenga la certeza y confianza de que lo propuesto es realmente una solución integral al conflicto; atendiendo principalmente sus causas y no solo sus efectos, siendo necesaria la participación activa de la sociedad en su conjunto. Para ello se requiere un cambio de pensamiento orientado hacia el desarrollo sostenible, buscando una nueva relación humanidad-naturaleza.

\begin{abstract}
The scope of Environmental Management in the public sector must be understood as the interaction of the technical-scientific and social-participatory. The first, conducting research leading to alternative solutions that are economically feasible and environmentally viable, in order to make the second has the certainty and confidence that the proposal is really a comprehensive solution to the conflict; mainly serving their causes and not only its effects, with the necessary active participation of society as a whole. This requires a change in thinking oriented toward sustainable development, looking for a new relationship humanity-nature.
\end{abstract}

\section{Introducción}

Cuando se discute el tema de los alcances de la Gestión Ambiental en la academia universitaria, en los sectores público y privado, en organizaciones no gubernamentales (ONG) y en las comunidades, es común apreciar diversas interpretaciones. La apertura a la interpretación de este concepto responde a la libre percepción que cada instancia asume - adopta según sus necesidades de atención o enfoque. Para un investigador en ciencias naturales, su enfoque puede discernir de otro investigador en ciencias sociales, y así mismo, los resultados de sus investigaciones pueden ser comprendidos de forma diferente según quien haga la lectura del trabajo, con diferentes interpretaciones según el contexto político, socio-ambiental, cultural, administrativo y empresarial, o cualquier otro que llegue a utilizar como marco de referencia.

Con el objetivo de analizar el concepto para comprender las implicaciones de la Gestión Ambiental en el sector público, en la I Parte de este documento se expusieron varias consideraciones que le permitieran al lector reflexionar sobre el tema, en consideración al desarrollo general de las culturas y su percepción del ambiente, así como la interpretación de varios autores que han trabajado el asunto, buscando su comprensión integral. En esta II Parte se expondrá una visión particular de lo que significa la Gestión Ambiental en el sector público, siendo ésta una propuesta conciliadora cuya motivación es inyectar mayor discusión para la evolución del paradigma de la Gestión Ambiental en la actualidad.

\section{Profundizar en la discusión de la Gestión Ambiental: de la transdisciplinariedad y complementariedad requeridas}

Desde un enfoque transdisciplinario y complementario de las ciencias naturales y sociales, o mejor concebido como lo socioambiental, los conflictos ambientales guardan estrecha relación con el tema de Gestión de Riesgo, el cual vincula lo que sucede en lo natural con lo acontecido (en buena medida) por las acciones antrópicas negativas. Una amenaza natural es de considerable atención cuando se evidencian situaciones de alta vulnerabilidad social. Por ello, educar para reducir el riesgo socioambiental requiere un proceso de Educación Ambiental 
(EA), con instructores académicos que asuman activamente su rol orientador en la formación del alumnado. Una actitud activa del modelo cívico que el educador debe seguir es lo que le permite al estudiante involucrarse a través del ejemplo de su orientador. En la medida en que el maestro o profesor se involucre activamente en los asuntos ambientales, "esto le permitirá entender y asumir la gestión del riesgo como una herramienta de la gestión ambiental para el Desarrollo Sostenible" (Alpízar, 2009, p. 68).

De la complementariedad analizada por Mardones (|99|) y su relación con el enfoque transdisciplinario de García (2006), es posible apreciar que las metodologías científicas y sus nuevas tendencias para los positivistas aceptan la comprensión de las ciencias sociales en sus fines y motivos ante el acontecimiento de los hechos, utilizando las herramientas de la explicación causal. Explicar por qué suceden esos hechos mediante la formulación teórica sujeta a verificación continua, sería un paso interesante siempre y cuando se diera su integración, con el fin de completar ese todo que conforman los hechos. Es así que los asuntos que interesan en las ciencias sociales y naturales no son independientes, ya que los unos afectan a los otros.

Como ejemplo de anterior, a) reflexiónese sobre el actual y evidente cambio climático por el calentamiento global, inducido en buena medida por la acción antrópica negativa del ser humano, en su afán de consumo energético a partir de fuentes de energía de difícil renovación en el tiempo (petróleo), más allá de la satisfacción de sus necesidades básicas, y b) las consecuencias socioambientales y culturales del terremoto de Haití del 12 de enero de 2010, por el brote de cólera que se convirtió en una epidemia (Notimex, 10 de noviembre 20 I0), debido al colapso de los sistemas sanitarios y la dispersión de los cadáveres descompuestos en las zonas afectadas y habitadas del país.

Bajo la visión del desarrollo de García (2006), la investigación multidisciplinaria surgida en la década de 1980 fue impulsada por instituciones de desarrollo apoyadas por la cooperación internacional. Lo multidisciplinario conceptualizado bajo el enfoque de investigación científica, considera varias disciplinas que atienden un problema con marco común. Aunque se inicie desde lo individual discipli- nario (objetivos diferenciados), su análisis se integra y vincula en un todo, atendiendo los diferentes aspectos del problema marco para ofrecer alternativas de solución integral.

Conceptualmente, lo transdisciplinario se asume como lo transversal de diferentes disciplinas y más allá de todas ellas, con la finalidad de comprender el mundo analizado, siendo su imperativo la unidad del conocimiento. Aunque García (2006) lo señala como un enfoque incipiente, la realidad es que en la práctica se hace más que necesario, debido a que los esfuerzos aislados (aparte de ser costosos) no son congruentes ni vinculantes a la hora de brindar alternativas viables de desarrollo o en la atención de los grandes conflictos socioambientales (ciencias sociales y ciencias naturales). Ello presupone un extraordinario proceso de comunicación entre los grupos (disciplinas) o, mejor aún, los elementos complementarios esperados por Mardones (199|). Dicho en lenguaje coloquial: "Cada quién en 10 suyo y todos en los mismo!". La sola mención de que todos los profesionales "estén en lo mismo" condiciona el asumir una actitud de mutuo respeto y una disponibilidad para constituir equipos de trabajo que atiendan una determinada problemática, siendo indispensable que cada integrante tenga una conciencia socioambiental que le permita enfocarse en el cumplimiento del objetivo, es decir, asumir el compromiso de ser parte de la solución integral.

Tener conciencia ambiental no es un asunto de conocer del tema (aspecto aprendido en la academia o por empirismo); se trata más bien de saber del tema (sabiduría que conjuga conocimiento, práctica, experiencia, intuición y destreza en su aplicación para obtener resultados positivos). La sensibilidad y la agudeza para analizar trasfondos sociales, políticos, económicos, culturales y ambientales se logra mediante un proceso reflexivo de autocrítica constructiva, donde solo es posible crecer asumiendo el compromiso personal de mejora continua. Luego de este proceso, es pertinente realizar críticas ante las situaciones disconformes, proponiendo cambios oportunos con justicia y equidad socioambiental, en concordancia con la ética, la moral y los valores positivos culturales que distinguen a cada localidad, con el fin de lograr el progreso.

Para Novo (2006), el progreso, cuando ocurre, es ante todo una conquista de la conciencia que 
precede a cualquier otro fenómeno material, es decir, lograr el sueño del desarrollo sostenible entendido como un proceso de transformación social requiere el rescate colectivo para recuperar lo abandonado, el saber perdido en la búsqueda del conocimiento y el conocimiento perdido cuando nos llenamos de información. Por ello, el autor señala que es necesario rescatar algunos principios tales como: la comprensión de la naturaleza y del planeta como algo finito, los límites de los sistemas ecológicos y sociales, el valor de la diversidad cultural, la sostenibilidad global mediante el respeto de los ciclos naturales, mayor eficiencia en los procesos y menor consumo de recursos, la necesidad de orientar la ciencia por criterios éticos, el valor de los sentimientos, las emociones y los afectos - "expulsados" en la visión moderna del mundo por el modelo racionalista-, el valor del conocimiento tácito y de las culturas originarias, la complementariedad persona-naturaleza, el valor de lo pequeño y lo descentralizado, la condición limitada de la ciencia y la tecnología, el principio de equidad en el reparto de los bienes naturales y de las conquistas científicas y tecnológicas, el valor de lo comunitario, su resistencia y su capacidad para afrontar perturbaciones, y otros. Se trata de principios que propician directrices para una vida más equilibrada desde la lógica ecológica y una sociedad más equitativa.

Construir una percepción del desarrollo sostenible a partir de las consideraciones anteriores, es sin duda una labor que le compete a lo interdisciplinario y lo complementario de las ciencias sociales y naturales; de forma individual sería casi imposible poder atender la complejidad del tema socioambiental, en atención a todos sus aspectos éticos, morales, racionales y por supuesto de sostenibilidad. Cuando ha existido ausencia de tal percepción, la respuesta tecnocientífica para tratar temas que afectan a la sociedad ha perdido validez ante la mirada crítica de los sectores sociales que no entienden su lenguaje, situación que hace necesaria la interpretación sociológica mediante técnicas asertivas de comunicación colectiva. Una parte es así, porque la comunidad debe hacer suyas las soluciones alternativas viables, a fin de que ellas puedan prosperar, y por otro lado, el uso de un lenguaje apropiado es un deber del científico y un derecho del ciudadano.
De esta forma, lo socioambiental no puede trabajarse exclusivamente con el encuadramiento de las ciencias naturales ni tampoco con el idealismo de las ciencias sociales. Se requiere de ambas para lograr la factibilidad y viabilidad de los proyectos que atiendan el objetivo de desarrollo sostenible. Por ello, debe darse un proceso transparente de negociación entre ambas ciencias para poder construir una adecuada gestión ambiental integral, que permita interrelacionar todos los aspectos socioambientales con la idea clara de propiciar el surgimiento de una nueva sociedad, es decir, una nueva cultura ambiental.

\section{Diferencia entre la gestión ambiental en el sector privado y el sector público}

De acuerdo con Negrão (2000), el factor determinante en la implementación de la Gestión Ambiental en el sector privado ha sido la presión ejercida por la opinión pública (sociedad) que impulsó las transformaciones en los paradigmas del desarrollo. En este sentido, la presión de la sociedad civil organizada ha permitido el eventual respaldo gubernamental a través de la reglamentación de las acciones para prevenir, reducir o mitigar la degradación ambiental empresarial.

Las medidas legales restrictivas, las nacionales y aquellas generadas en las normas y tratados internacionales, han aumentado considerablemente con el fin restringir la comercialización de productos cuyo proceso es contaminante en los países que perjudican el ambiente. En el sano comportamiento ambientalmente empresarial, no se sigue una regla para todos, persistiendo los factores de costos y mercado como los determinantes en el lucro y el cambio en el comportamiento empresarial. Es así que:

"(...) persisten los casos en los que la relación entre empresa y medio ambiente todavía es un asunto ignorado, o no es bienvenido pues la protección ambiental todavía es entendida solamente como costos adicionales que perjudican la competitividad y el crecimiento de la empresa". (Negrão, 2000, p. 31).

Los principales enfoques de la gestión ambiental en el sector empresarial responden a la imposición de la problemática ambiental durante las transformaciones de los paradigmas. En la década de 1970, la estrategia de las empresas en los países desarro- 
llados fue "contaminar y después descontaminar", o "la solución para la contaminación es la dilución", es decir, cumplir con las normas y evitar accidentes, pero siempre buscando maximizar el lucro en el corto plazo (economía de frontera), siendo inviable por el incremento en el volumen de contaminantes y la saturación del ambiente como receptor. Posteriormente, con el paradigma de la Protección Ambiental (control de la contaminación), se utilizan tecnologías "end of pipe" (o de fin de línea) para reducir las emisiones, resultando incompatibles la responsabilidad ambiental empresarial y la maximización del lucro. Se desarrolla la reingeniería en los procesos para disminuir costos en producción y minimizar la generación de residuos, con resultados favorables a nivel energético, siendo una buena respuesta al paradigma de la gestión de recursos. En la década de 1980, el sector empresarial en los países desarrollados lo asumió como una necesidad de supervivencia, pasando de agentes reactivos o defensivos a previsores y protectores, atendiendo la evolución de la conciencia ecológica.

Los principales instrumentos de la gestión ambiental empresarial o en el sector privado son de tipo preventivo, correctivo, de remediación y/o proactivas, según la fase en la que se implementen. Ante todo, la gestión realizada por el sector privado se orienta fundamentalmente a mantener una imagen de cumplimiento de las normas ambientales en el país donde opera, y no más allá de ellas. Además de que el asunto ambiental se ha utilizado como un factor de competitividad con otras empresas dedicadas a la oferta de productos similares, con un afán de capturar un mayor número de demandantes de sus productos, de manera que el asunto ambiental se usa como un medio de ganancia.

En el caso del sector público, la situación es muy diferente ya que este se rige por la política ambiental nacional, que establece principios y directrices de acatamiento obligatorio para la institucionalidad, en el marco de un sistema de gestión ambiental para el sector público. Los municipios también son los llamados a ordenar y gestionar los distintos ámbitos que afectan lo urbano-ambiental como un componente del desarrollo sostenible en las ciudades, siendo a nivel local el lugar donde se expresan más claramente los problemas ambientales que aquejan o inciden en el ciudadano (Reyes, 20l0). En este sentido, el municipio es el principal órgano articulador de demandas y soluciones ciudadanas, por lo cual la creación de una política ambiental y una efectiva acción de su gestión ambiental municipal se convierten en una meta para hacer sostenible el desarrollo local del territorio municipal.

La gestión ambiental municipal se constituye por un conjunto de acciones tendientes al logro de la máxima racionalidad en el proceso de decisión relativo a la conservación, defensa, protección y mejora del ambiente, a partir de un enfoque interdisciplinario y complementario. Para ello requiere instrumentos de gestión que le permitan actuar desde el ámbito global hasta el local, involucrando a la sociedad en el cumplimiento de los objetivos ambientales. Tales instrumentos de gestión se resumen, de acuerdo con Reyes (20I0), en principios (cuadro I) y criterios (cuadro 2) que son la base para comprender el alcance del actuar institucional a favor del desarrollo sostenible en las comunidades.

Como es comprensible, la única coincidencia entre la gestión ambiental que se desarrolla entre el sector privado y el público se da en la prevención de la contaminación. Sin embargo, para el primero se trata de un asunto de competitividad comercial y de cumplimiento de las normas impuestas ante la presión social, y para el segundo es una obligación de eficiencia y eficacia en el cumplimiento de su deber de ser agente del Desarrollo Sostenible a favor de lo socioambiental' en el espacio urbano. La carga de trabajo para cumplir con los principios y criterios de la Gestión Ambiental señalados por Reyes (20l0) requiere del trabajo interdisciplinario y complementario de equipos de trabajo que en buena medida puede ofrecer el sector público, y por supuesto las fuertes inversiones de recursos públicos necesarios para realizar los estudios de viabilidad y factibilidad socioambiental y su posterior ejecución. Como queda en evidencia, un verdadero proceso de Gestión Ambiental para el Desarrollo Sostenible que permita la construcción de una cultura que asegure el aumento del nivel y calidad de vida de los ciudadanos, solo es posible desde la administración pública ya que su razón de ser es la satisfacción de las necesidades comunales, misma que en su mayoría reclama por un cambio de paradigma que le permita establecer una nueva persecución en la relación

I Para ampliar el tema de eficiencia administrativa, se recomienda el artículo de Johnson, H. 2009. La Certificación en el Sector Público: una necesidad de competencia o un deber de eficiencia administrativa. Disponible en www.uned.ac.cr/sep/recursos/revista/ documents/4-lacertificacionenelsectorpublico-HelmuntJonhson.pdf 
Cuadro I. Principios de sostenibilidad, justicia social y toma de decisiones en la Gestión Ambiental en el sector público.

\begin{tabular}{|c|c|c|}
\hline Principio & Nombre & Consiste en: \\
\hline \multirow{7}{*}{ 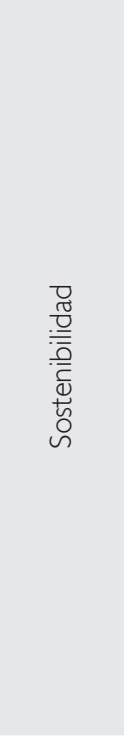 } & Prevención & $\begin{array}{l}\text { Prevenir la degradación y la contaminación antes de que ocurra, más que limpiar o } \\
\text { solucionar el daño posterior. }\end{array}$ \\
\hline & Precaución & $\begin{array}{l}\text { Ante la dudas acerca de las consecuencias ambientales de una acción, la falta de evidencias } \\
\text { científicas no se deberá usar como justificativo para postergar la adopción de las medidas } \\
\text { necesarias para prevenir los presuntos daños ambientales al medio físico, biótico y humano. }\end{array}$ \\
\hline & Sustitución & $\begin{array}{l}\text { Las sustancias que constituyen un riesgo para la salud y el ambiente deberán ser sustituidas } \\
\text { por otras con mejores cualidades para la salud y el ambiente. }\end{array}$ \\
\hline & $\begin{array}{l}\text { Carga inversa de la } \\
\text { prueba }\end{array}$ & $\begin{array}{l}\text { El contaminador asume la responsabilidad de probar ante las autoridades y la sociedad que } \\
\text { sus actividades no producen perjuicio alguno a la salud o al ambiente. }\end{array}$ \\
\hline & Contaminador pagador & $\begin{array}{l}\text { Los potenciales generadores de contaminación son los responsables de los costos de } \\
\text { prevención y de las medidas de remediación y limpieza. No significa autorización para } \\
\text { contaminar. }\end{array}$ \\
\hline & $\begin{array}{l}\text { Requisito del } \\
\text { conocimiento }\end{array}$ & $\begin{array}{l}\text { Quien desarrolle actividades que pueden tener un impacto en la salud o en el ambiente } \\
\text { debe tener necesariamente el conocimiento para prevenir los efectos perjudiciales que se } \\
\text { deriven de esa actividad. }\end{array}$ \\
\hline & $\begin{array}{l}\text { Capacidad de carga de } \\
\text { los ecosistemas }\end{array}$ & $\begin{array}{l}\text { Se interpreta como un límite de absorción de la contaminación y de la explotación } \\
\text { humana sin que afecte su capacidad de regeneración natural. }\end{array}$ \\
\hline \multirow{4}{*}{ 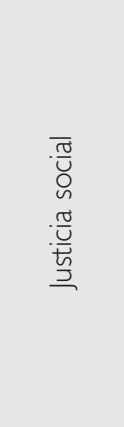 } & Derechos & $\begin{array}{l}\text { Reconocimiento y aplicación en cualquier ámbito de la Gestión Ambiental; todos los } \\
\text { derechos de la sociedad reconocidos nacional e internacionalmente. }\end{array}$ \\
\hline & Cooperación & $\begin{array}{l}\text { Todos los actores sociales tienen el derecho de comprometerse en la formulación e } \\
\text { implementación de los procesos de planificación de la gestión ambiental. }\end{array}$ \\
\hline & $\begin{array}{l}\text { Acceso justo a } \\
\text { los recursos y } \\
\text { representación honesta }\end{array}$ & $\begin{array}{l}\text { Gestión del acceso y control sobre los recursos al interior y entre comunidades, así como } \\
\text { también entre géneros, grupos socioculturales y generacionales. }\end{array}$ \\
\hline & $\begin{array}{l}\text { Satisfacción necesidades } \\
\text { humanas }\end{array}$ & $\begin{array}{l}\text { Se debe avalar el desarrollo social y económico que haga posible que la sociedad satisfaga } \\
\text { las necesidades humanas básicas de vivienda, empleo, educación, servicios de salud, agua } \\
\text { limpia y alimentación, mejorando las condiciones de vida mientras se respeta el ambiente. }\end{array}$ \\
\hline \multirow{5}{*}{ 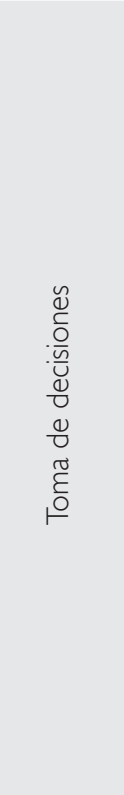 } & Democracia & $\begin{array}{l}\text { Garantía del control al gobierno local por parte de los residentes a través de sus } \\
\text { representantes electos. La comunidad en general controla el cómo se deben gastar los } \\
\text { recursos locales proveniente del pago de tasas, impuestos y servicios locales. }\end{array}$ \\
\hline & Participación comunitaria & $\begin{array}{l}\text { Incluye la definición de acciones, información, diseminación y preparación social para la } \\
\text { comunidad y sector afectado, antes de la aplicación de los procedimientos y del proceso } \\
\text { de retroalimentación que incluye el seguimiento de las acciones y evaluación de sus } \\
\text { impactos. }\end{array}$ \\
\hline & Subsidiariedad & $\begin{array}{l}\text { Las decisiones del gobierno local, frente a acciones de desarrollo, deben ser tomadas lo } \\
\text { más cerca posible de la gente directamente afectada. La Gestión Ambiental debe apoyar } \\
\text { permanentemente aquellas estructuras administrativas desconcentradas que carecen o } \\
\text { son débiles en materia de prevención y control ambiental, mientras asumen su rol con } \\
\text { efectividad. }\end{array}$ \\
\hline & Coherencia & $\begin{array}{l}\text { Los mecanismos de toma de decisiones y su validación política por el máximo organismo } \\
\text { del gobierno local deben ser claramente definidos. }\end{array}$ \\
\hline & $\begin{array}{l}\text { Transparencia y } \\
\text { rendición de cuentas }\end{array}$ & $\begin{array}{l}\text { El proceso y los parámetros para la toma de decisiones deben ser claros, explícitos } \\
\text { y públicos. El gobierno local debe elaborar informes escritos donde se detallen los } \\
\text { potenciales beneficios y desventajas de alguna medida; las memorias del debate y las } \\
\text { decisiones deben estar disponibles para el conocimiento de la opinión pública (rendición } \\
\text { de cuentas). }\end{array}$ \\
\hline
\end{tabular}

Fuente: Reyes, 20 10. Documento Integrador C. DOCINADE, pp. I-3. 
Cuadro 2. Criterios de la Gestión Ambiental desde el ámbito local

\begin{tabular}{l|l|l|}
\hline \multicolumn{1}{|c|}{ Criterio } & \multicolumn{1}{c}{ Considera: } \\
\hline $\begin{array}{l}\text { La investigación, la } \\
\text { información y la educación }\end{array}$ & $\begin{array}{l}\text { Mecanismos orientados a identificar las acciones de origen antrópico y natural que requieren de una } \\
\text { atención prioritaria y planificada, así como a la toma de conciencia por parte de la comunidad para } \\
\text { que asuma su rol protagónico en los sistemas de gestión ambiental y desarrollo sustentable. }\end{array}$ \\
\hline El ordenamiento territorial & $\begin{array}{l}\text { Herramienta moderna de planificación que oriente una distribución equilibrada y ordenada de las } \\
\text { actividades vinculadas con el crecimiento urbano y el ambiente. }\end{array}$ \\
\hline La participación ciudadana & $\begin{array}{l}\text { Aspecto estratégico de la planificación para la Gestión Ambiental, orientada a incorporar las } \\
\text { responsabilidades de la sociedad en los procesos de toma de decisiones para la definición de su } \\
\text { modelo de desarrollo. }\end{array}$ \\
\hline $\begin{array}{l}\text { El monitoreo y vigilancia de } \\
\text { la calidad ambiental }\end{array}$ & $\begin{array}{l}\text { Aspectos de la Gestión Ambiental determinantes para iniciar acciones de prevención, control y } \\
\text { remediación ambiental, con el fin de alcanzar los objetivos de calidad ambiental y conservación de } \\
\text { los recursos naturales. }\end{array}$ \\
\hline $\begin{array}{l}\text { El control y la fiscalización } \\
\text { ambiental }\end{array}$ & $\begin{array}{l}\text { Aspectos concretos del ejercicio de la autoridad ambiental orientados a la aplicación y cumplimiento } \\
\text { efectivo de la normativa ambiental. }\end{array}$ \\
\hline La cooperación & $\begin{array}{l}\text { Mecanismo estratégico para lograr soporte y apoyo para la implementación de planes y programas } \\
\text { concebidos, así como para desarrollar la gestión ambiental de manera armónica y consecuente con } \\
\text { las políticas ambientales nacionales. }\end{array}$ \\
\hline $\begin{array}{l}\text { y coordinación } \\
\text { interinstitucional }\end{array}$ & $\begin{array}{l}\text { Instrumentos que facilitan la transparencia de la gestión ambiental vinculando de manera más directa } \\
\text { a los actores sociales y al gobierno local y garantizando la rendición de cuentas hacia la comunidad. }\end{array}$ \\
\hline y difusión de información &
\end{tabular}

Fuente: Reyes, 20 I0. Documento Integrador C. DOCINADE, p. 3.

humanidad-naturaleza. Resumiendo los comentarios de Novo (2003), es pasar del antropocentrismo etnocentrista y androcéntrico al biocentrismo con su enfoque ético centrado en la vida.

\section{Conclusión}

Es así que la correcta GestiónAmbiental,en su estrecha vinculación con el quehacer de las ciencias sociales y ambientales, debe ser entendida apropiadamente como la interacción entre lo técnico-científico con lo social-participativo. Ciencia y tecnología que actúen en función de la realidad biofísica en respuesta a los problemas particulares que se presenten en una región determinada, y proceso de participación social activo que se apropie de la mejor alternativa de solución que ofrecen los primeros; siempre y cuando tales alternativas conjuguen los aspectos sociales, económicos, políticos, culturales y ambientales a favor del Desarrollo Sostenible, propiciando la construcción de una mejor relación socioambiental con justicia y equidad entre las partes.

Desde esta percepción de la Gestión Ambiental, es comprensible para el sector público que las soluciones a los grandes conflictos ambientales a nivel local y regional no pueden resolverse con los pequeños aportes surgidos de investigaciones desvinculadas de la interacción técnico-científica y social-participativa, que solo contribuyen a solucionar algún efecto más no la causa del problema. Ello no implica que el aporte científico, tecnológico, sociológico, económico, político y hasta normativo no goce de validez, sin embargo, los esfuerzos aislados que no tienen como meta común la solución de un conflicto socioambiental no logran encajar como piezas de un rompecabezas. En la tarea de tratar de unificar los aportes individuales, normalmente se requiere mayor esfuerzo en su adaptación, traducido en mayor inversión de recurso humano y recursos públicos. Dicha situación es en sí misma un conflicto, cuando se debe convencer a los tomadores de decisión (políticos) que responden a una comunidad donde su credibilidad en el sector público no expresa el mejor apoyo ante el esfuerzo por implementar soluciones a los grandes problemas socioambientales. Ello es comprensible en razón de las lecciones aprendidas, cuando se han tratado de encontrar soluciones a los problemas de forma desvinculadas, por lo que éstas no contribuyen más que a agravar la situación conflictiva. 
La construcción de una cultura socioambiental correcta, que atienda la demanda de un nuevo paradigma en la relación humanidad-naturaleza, ciertamente requiere mayores espacios de debate desde lo técnico-científico y lo social-participativo, para juntos construir y contribuir al Desarrollo Sostenible. Esto permitirá tener claro cuál es la Costa Rica que queremos para nosotros, o mejor aún, la Centroamérica que aspiramos ser en este nuevo milenio; una región efectivamente ordenada, una conciencia ambiental propia que responda a nuestras raíces culturales y una visión filosófica con ética, moral, justicia y equidad socioambiental, algo que efectivamente podamos heredar a las futuras generaciones, jsi es que para entonces queda algún planeta que heredarles!

\section{Bibliografía}

Alpízar, Ma. L. (2009). Educación y Reducción de Riesgos y Desastres en Centroamérica: Gestión del Riesgo. [Versión
Digital PDF]. Obtenido desde: http://www.ceducar.info/ coleccion_formacion/volumen50.pdf

Mardones, J.M. (|99|). Filosofía de las ciencias humanas y sociales: nota histórica de una polémica incesante. En: Filosofía de las ciencias humanas y sociales: materiales para una fundación científica, (pp. 19-42). Obtenido desde: http://aminarashida. files.wordpress.com/20 I I/06/mardones-j-m-filosofia-de-lasciencias-humanas-y-sociales-pdf-june-26-20 I 0-3-34-am$|58 \mathrm{k}| . p d f$

Negrão, R. (2002). Gestión Ambiental. En: UNESCO (ed.). II curso internacional de aspectos geológicos de protección ambiental: notas de clases [Versión Digital PDF]. Montevideo, Uruguay. pp. 27-35. Obtenido desde: http://unesdoc.unesco. org/images/0016/001631/163153s.pdf

Notimex (20 I0, nov. 10). ONU busca contener epidemia en Haití. En: El Universo.mx. Obtenido desde http://www.eluniversal. com.mx/notas/722521.html

Novo, M. (2003). La Educación Ambiental: bases éticas, conceptuales y metodológicas (3a. ed). Madrid: Universitas.

Novo, M. (2006). El Desarrollo Sostenible: su dimensión ambiental y educativa. Madrid: España: Pearson \& Prentice Hall.

Reyes, L.B. (2010). Documento integrador C: Material integrado para su uso didáctico. Cuautitlán, México: DOCINADE. 